\title{
Ipilimumab trotz Autoimmunerkrankung?
}

\author{
Patienten mit fortgeschrittenem \\ Melanom können unter besonderen \\ Kautelen offenbar auch bei Auto- \\ immunerkrankung Ipilimumab \\ erhalten.
}

W ird die hemmende Wirkung des Immuncheckpointmoleküls CTLA(„Cytotoxic T Lymphocyte Antigen")-4 durch den Antikörper Ipilimumab aufgehoben, können T-Zellen wieder verstärkt gegen Tumorzellen vorgehen, was beim fortgeschrittenen Melanom zu einem längeren Überleben führt. $\mathrm{Da}$ aber gesundes Gewebe ebenfalls Ziel der T-Zellen werden kann, gibt es auch unerwünschte Effekte wie Kolitis oder Hypophysitis. Patienten mit Autoimmunerkrankungen (AIE) sind daher aus klinischen Studien mit Ipilimumab ausgeschlossen und erhalten es auch im Alltag nur selten. US-amerikanische Ärzte sahen sich jetzt rückblickend 30 solcher $\mathrm{Pa}$ - tienten mit rheumatoider Arthritis (RA, $\mathrm{n}=6$ ), Psoriasis (5), chronisch-entzündlicher Darmerkrankung (6), systemischem Lupus erythematodes (2), multipler Sklerose (2) und Autoimmun-Thyreoiditis (2) an. 13 standen deswegen zu Beginn der Ipilimumab-Therapie unter Immunsuppression.

Exazerbationen der AIE traten bei 8 Patienten (27\%) binnen 3 Tagen bis 7 Monaten nach Beginn der IpilimumabTherapie auf, meist erneute oder verstärkte Manifestationen früherer Symptome. Alle waren mit systemischem Prednison (6-mal 5-30 mg/d, 2-mal $1 \mathrm{mg} / \mathrm{kg} / \mathrm{d}$ ) behandelbar.

Durch Ipilimumab induzierte immunologische Nebenwirkungen vom Grad $3-5$ betrafen 10 Patienten (33\%; 5-mal Kolitis, 3-mal Hypophysitits, je 1-mal Thyreoiditis bzw. akutes Glaukom) nicht mehr als in klinischen Studien. Bei 7 Patienten waren diese Nebenwirkungen durch Steroide reversibel, 2 benötig- ten Infliximab. Eine AI-Kolitis bei einem Psoriasis-Patienten endete tödlich, wohl weil er erst eine Woche nach Beginn der Diarrhö zum Arzt ging. 10\% der Patienten erfuhren sowohl eine Verschlechterung der AIE als auch immunologische Nebenwirkungen, $50 \%$ der Patienten blieben von beidem verschont.

Objektiv sprachen 6 Patienten $(20 \%)$ an, einer (mit Exazerbation der RA und Hypophysitis) vollständig und seit mittlerweile fast 2 Jahren. Die mediane Gesamtüberlebenszeit betrug 12,5 Monate.

Fazit: Die Forscher schließen daraus, "dass eine Ipilimumab-Therapie bei vielen Patienten mit bestehender AIE nach ausführlicher Aufklärung und unter engmaschiger Überwachung in Betracht gezogen werden kann" - aber wohl nicht für Patienten mit besonders schwerer Grunderkrankung. Beate Schumacher

Johnson DB et al. Ipilimumab Therapy in Patients With Advanced Melanoma and Preexisting Autoimmune Disorders. JAMA Oncol. 2016;2(2):234-40.

\section{Durchmesser und Tiefe beim MCC wichtig}

\section{In 24-31\% der Fälle sind bei Diagnose des Merkelzellkarzinoms (MCC) bereits regionale Lymphknoten befallen. Welche Faktoren beeinflussen das Risiko für einen positiven Sentinellymphknoten (SLN) und ein kürzeres Überleben?}

In den meisten Leitlinien wird empfohlen, bei lokalisiertem MCC eine Biopsie des SLN durchzuführen, um einen etwaigen regionalen Lymphknotenbefall erkennen und therapeutisch darauf reagieren zu können. Retrospektiv wurde untersucht, welche Faktoren den SLN-Status bzw. das Überleben vorhersagen. Die Analyse stützte sich auf die Daten von 375 Patienten mit MCC, die zwischen 1988 und 2011 am Moffitt Cancer Center in Tampa, FL/USA, behandelt worden waren. Sie wurden in 5 Gruppen unterteilt: 1. negativer SLN-Status, 2. positiver SLNStatus, 3. klinisch nodal negativer Befund ohne SLN-Biopsie, 4. regionaler Lymphknotenbefall mit unbekanntem Primärtumor, 5. primäres MCC mit klinisch evidentem regionalem Lymphknotenbefall. $70 \%$ der Patienten waren Männer, das mediane Alter lag bei 75 Jahren. Die häufigste Lokalisation des MCC war die Kopfund Halsregion (45\%), gefolgt von den oberen Extremitäten ( $23 \%)$, den unteren (13\%) und dem Körperstamm (8\%).

Der mediane Tumordurchmesser betrug $1,5 \mathrm{~cm}(0,2-12,5 \mathrm{~cm})$, die mediane Tumortiefe 4,8 mm (0,3-45 mm). Bei 59 von 191 Patienten (31\%) war eine SLNBiopsie positiv. Ein solcher positiver Status war mit einem größeren Durchmesser des Primärtumors und tieferem Einwachsen assoziiert ( $p=0,007$ bzw. 0,017), nicht jedoch mit Alter bzw. Geschlecht.

154 Patienten verstarben, davon $45 \%$ infolge des MCC. Ein ungünstigeres Gesamtüberleben war mit Immunsuppression, größerem Tumordurchmesser und größerer Eindringtiefe assoziiert ( $p=0,007,0,003$ bzw. 0,025). Patienten mit negativem SLN überlebten median 85 Monate im Vergleich zu 60 Monaten bei Patienten mit positivem SLN-Befund; der Unterschied zeigte einen Trend zur Signifikanz (Log-rank $\mathrm{p}=0,065)$.

Das krankheitsspezifische Überleben war bei Patienten mit negativem SLN-Status am besten, bei jenen mit primärem MCC und klinisch evidentem regionalem Lymphknotenbefall am schlechtesten (p $=0,018$ ). Die krankheitsspezifische 5-Jahres-Todesrate betrug in der SLN-positiven Gruppe $26 \%$, in der SLN-negativen Gruppe $14,8 \%$.

Fazit: Beim MCC sind größerer Durchmesser und Eindringtiefe des Primärtumors unabhängige Prädiktoren für einen positiven SLN, ein schlechteres Gesamt- und krankheitsspezifisches Überleben. Die Eindringtiefe sollte Teil der histopathologischen Routinebefundung sein.

Kathrin von Kieseritzky

Smith FO et al. Both tumor depth and diameter are predictive of sentinel lymph node status and survival in Merkel cell carcinoma. Cancer. 2015;121(18):3252-60. 